\title{
Monitoring the Impact of Solution Concepts within a Given Problematic
}

\author{
Denis Cavallucci, François Rousselot, and Cecilia Zanni \\ 24, Boulevard de la Victoire 67084 Strasbourg Cedex, France \\ \{Denis.Cavallucci, François.Rousselot,Cecilia Zanni, \\ denis.cavallucci\} ainsa-strasbourg.fr
}

\begin{abstract}
It is acknowledged that one of the most critical issues facing today's organizations concerns the substantial leaps required to methodologically structure innovation. Among other published work, some suggest that a complete rethinking of current practices is required. In this article, we propose a methodology aiming at providing controlled R\&D choices based on a monitoring of the impact Solution Concepts provoke on a problematic situation. Initially this problematic situation is modeled in a graph form, namely a Problem Graph. It has the objective to assists R\&D managers when choosing which activities to support and bring them concrete arguments to defend their choices. We postulate that by improving the robustness of such approaches we help deciders to switch from intuitive decisions (mostly built upon their past experiences, fear regarding risks, and awareness of the company's level of acceptance of novelties) to thoroughly constructed inventive problem solving strategies. Our approach will be discussed using a computer application that illustrates our hypothesis after being tested in several industrial applications.
\end{abstract}

Keywords: TRIZ, Solution Concepts, Problem graph, contradictions, R\&D choices.

\section{Introduction}

Problem Solving is stated as one of the most universal and prevalent thinking activity and is nowadays more than ever a need for engineers evolving in R\&D departments of innovative firms [1]. It concerns engineers from both small and medium enterprises so as large scale corporations and this mankind capacity is claimed to be "the most important aspect of any job" [2]. Since inventive thinking abilities are specifically addressed when in inventive design, the arising need to manage their efficiency becomes unavoidable in innovation era.

In this context, TRIZ arrival on the industrial scene has been felt by most individuals as a potential solution to provide a structured approach to what was previously unstructured [3]. Nevertheless, and due to the difficulties to acquire sufficient competences in its practice for obtaining results, TRIZ has been stored in companies at the level of "creativity-like" technique and often replace an inefficient classical brainstorming. This phenomenon is even increased with the arrival of Computer tools based partially on TRIZ (sometimes named CAI tools) claiming to be a solution having successfully translated the theory into an operational procedure [4]. 
At such a point, we forgot that TRIZ was also the major theory of a wider research framework aiming at explaining Inventor's behavior. In our initial research activities, we stated on TRIZ limits and initiate works for identifying bottlenecks of its evolution. One of them consists in addressing complex and multidisciplinary situation using problem representation means. This first step highlighted in our findings that prior to engage Inventive Problem Solving Activities using TRIZ techniques, there must be a reduced area to focus on. In this reduced area, contradictions may be disclosed and clearly represent all inventive problems still unsolved and arising within the life of an artifact's evolution. Another of our findings led to a new formalism to classify contradictions in "clouds" for being able to chose which one was the most appropriate to be solved [5].

In the present article, we will focus in particular on a feedback between solutions concepts and problems in order to postulate that a specific solution concept is more suitable for impacting problems. This can be achieved in using, as an intermediary element, contradictions as a link between solution concepts and problems. As a result each solutions concept's impact on problems can be monitored and assist R\&D decider in his necessity to chose the most suitable investment in terms of engineering efforts among all possible variants.

\section{Gathering, Formulating and Answering to Problems}

\subsection{Representing and Chosing a Problem}

As we have already presented, complex situations requires advanced methodologies (often computer assisted) to map initial situations. Our approach derivated from what we have understood from OTSM [6], features a knowledge representation layer in which problems are described through a graph. In addition to this representation, known partial solutions may complete the domain understanding and serve our analysis to target the most appropriate problem to be further described in the remaining part of the methodology. Therefore, the problem is like an entrance point for better impacting on the graph, after the hypothesis that this problem can be somehow considered as a"key problem".

We automated, in our prototype software TRIZAcquisition V3.32, the targeting of such a problem in highlighting the first problem of the longest chain of problems when this chain is not influenced by the presence of a partial solution.

This has obviously not as objective to restric the overall problematic to the chosen problem but to enter into a further detailed description through a promizing angle. We assume the angle to be promizing based on our assumption that the first problem to be solved of the longest chain of problems, if solved, will remove all remaining problems of the chains it is related to, until it is not disturbed by the presence of a partial solution. As a consequence, we can advocate in the case illustrated figure 1, that if Problem 12 is solved, it has the potentially highest impact on our graph reduction.

At this point we are not sure about the real impact of problem 12 on the graph reduction, nevertheless this entrance point is necessary to further detail our problem statement and this choice has at least, highlighted the best possible candidate taking into consideration the fuzzy level of description we have. 


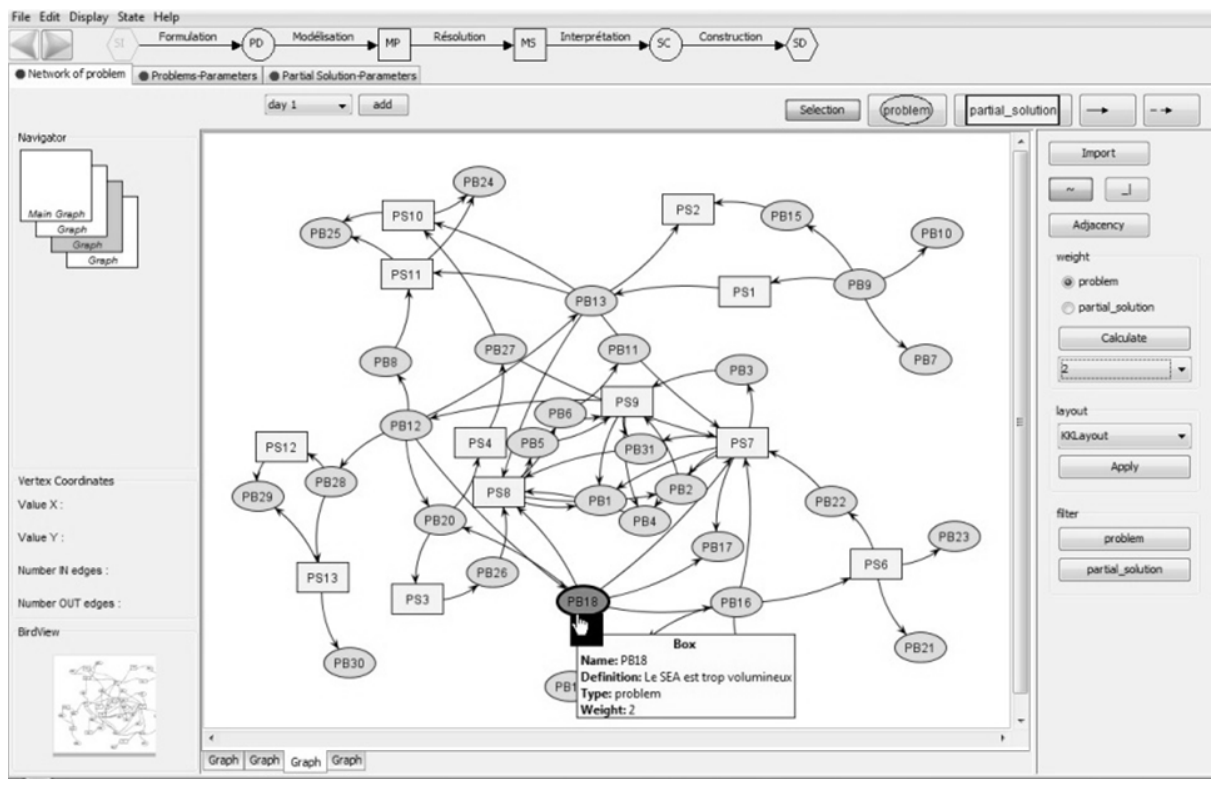

Fig. 1. Problem graph and its key problem entrance

\subsection{Translating Problems and Partial Solutions into Contradictions Components}

After entrance problem choice, no evident links are made between our network of problems and what we need in terms of data for engaging a solving process. What we know is that we need parameters to be disclosed from the situation in order to populate our templates of contradictions. These parameters are of two orders (evaluation or action). From our experiences in questionning experts, problems are tightly linked with evaluation parameters (EP) while partial solutions result more in acting parameters (AP) (see figure 2). As a result, our methodology includes a step for drawing these links and formulating the derivated set of parameters.

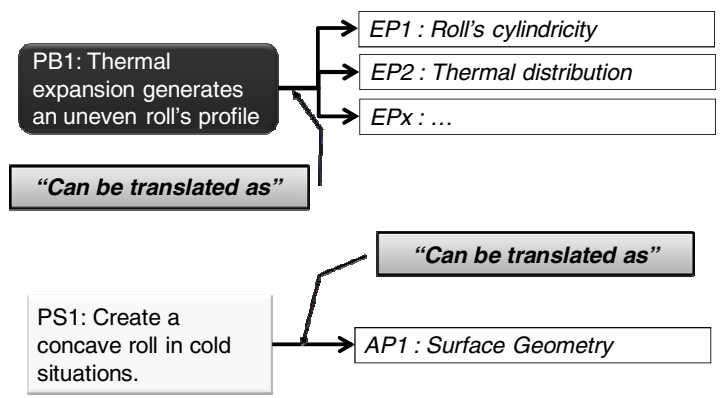

Fig. 2. Linking problem graph and contradiction components 
Even if the remaining part of the study may modify our first vision of the problem (therefore of the parameters) we shall keep the links created between problems and parameters for a relevant forthcoming measurment of the impact of concepts of the problem network.

\subsection{Constructing the Contradictions Cloud}

This phase is more related to what TRIZ has brought to Engineering Design: the concept of contradiction and the concept of Laws of engineering systems evolution. As it is presented in [7], there is a need at this stage of the methodology to disclose all contradictions inherent to our problematic and highliglt the most appropriate ones to be engaged in the solving process. The result of such a sequence of phases is a contradiction cloud (figure 3) as a support for the decision to solve one or several contradictions using tools of classical TRIZ.

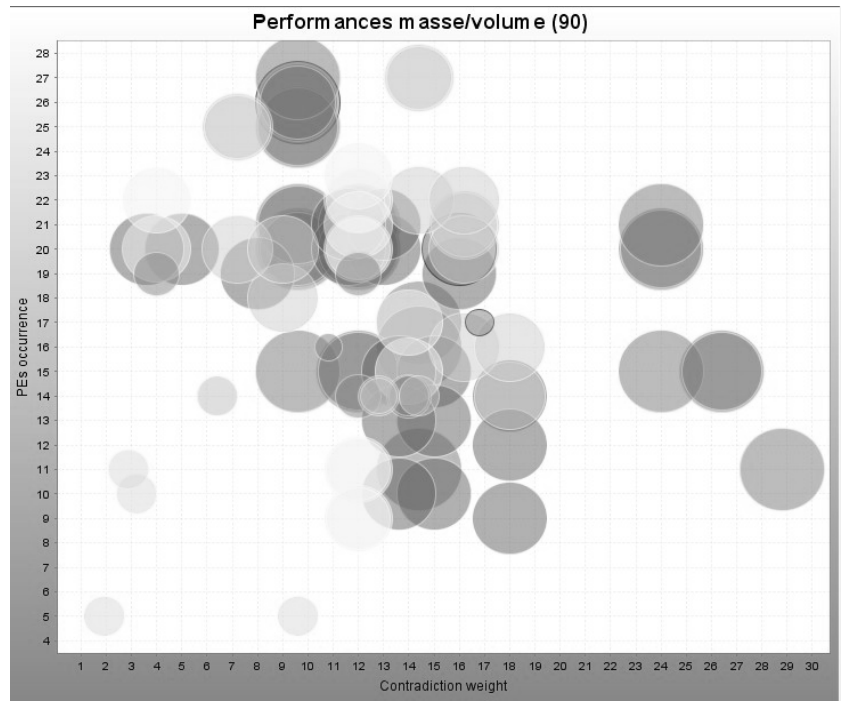

Fig. 3. Contradiction clouds and chosen TC

\subsection{Key Contradiction Solving and Solution Concepts Building}

When a subset of contradiction is chosen, classical tools of TRIZ can play their role and assist the team for generating solution concepts. Here we shall remind that a solution concept (SCX) result from the interpretation of a TRIZ knowedge base element (Inventive principles, Inventive Standards, Separation principles, physical effect ...). As these solution concepts are following TRIZ philosophy, they can only be kept (recorded for the rest of the study) if they quantitatively provoke the progress of each evaluating parameters in the appropriate direction (in oposition to resulting from a compromize). Generating inventive solution concepts is rather classical in TRIZ, they will not be considered within this paper. 


\subsection{Correlation Phase between Solution Concepts and Problems}

After solution concepts generation, we now possess a list of SCs and we want to evaluate their impact on our initial network of problems. At this stage, only the recorded links $\mathrm{PB} \rightarrow \mathrm{EP}$ can play this role. Nevertheless, throughout the study, EPs might have evolved in formulation, being erased or changed. Therfore it is necessary to refresh these links and complete all possible relations between EPs and SCs.

The fact that we want to highlight the relations for measuring the impact bring us another problem: How to rank the impacts and indicate the relevance of our assumptions up to a given robustness of our claims?

Our proposal in this regard is to use both Pugh's approach of qualitative evaluation and statistics [8]. For Pugh's approach, six levels of evaluation of links have been disclosed and must be used by several designated experts for linking SCs to EPs. On a practical point of view we generate tables automatically from our prototype of software and send each table to be filled to each expert.

In order to claim that their assumptions have a certain robustness (confidence level like) we employed a Statistical Approach in asking each expert to independently fill their grid based on common rules stated before to all expert evaluating. As a result, if more than 3 experts out of 4 have strongly qualifyed a link (more than 2 on a scale going from -3 to 3 ) without discussing to each other, we attributed a high level of confidence to such situations. If using the same principle more than 2 experts out of 3 have qualifyed the link above 1 on the same scale, we attribute a medium level of confidence to such situations.

After receiving 2 grids filled independetly by each expert, we translated their answers in links. The first grid is linking EPs to PBs while the second is linking SCs and EPs (therefore Contradictions). As a result, we can monitor the impact of an SC to a set of PBs. The result of 3 expert's evaluations on a case study gave us the following graph (figure 4):

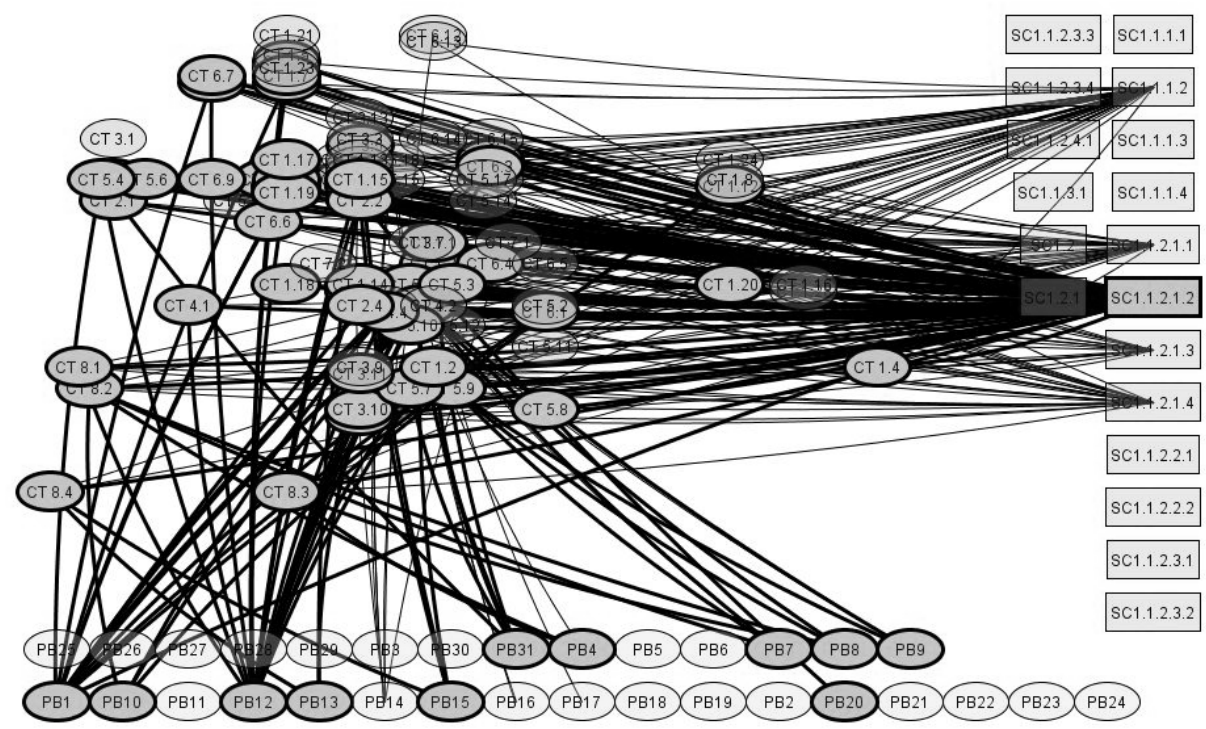

Fig. 4. Links of a medium confidence level from Solution Concepts to Problems 


\subsection{Scenarios Building and Display}

Each solution concept's impact can now be visualized through several levels of confidence. As we have seen in the previous praragraph, the lower the level of confidence is, the more links appears and impact more problems. All impacted problems are therefore subjected to dissapear since they will be hypothetically be solved. As a result, the problem graph will be reduced to the set of remaining problems (unsolved ones). Figure 5 is illustrating the impact of SC1.1.2.1.2 on our initial problems graph. Here, we can employ the term "shrinkage" of the problem graph for a specific SC.

After testing each SC at various confidence levels, project leaders can evaluate the amplitude of the impact of their ideas on the problem graph and decide which one (or which set of) shall be engaged in developpment.

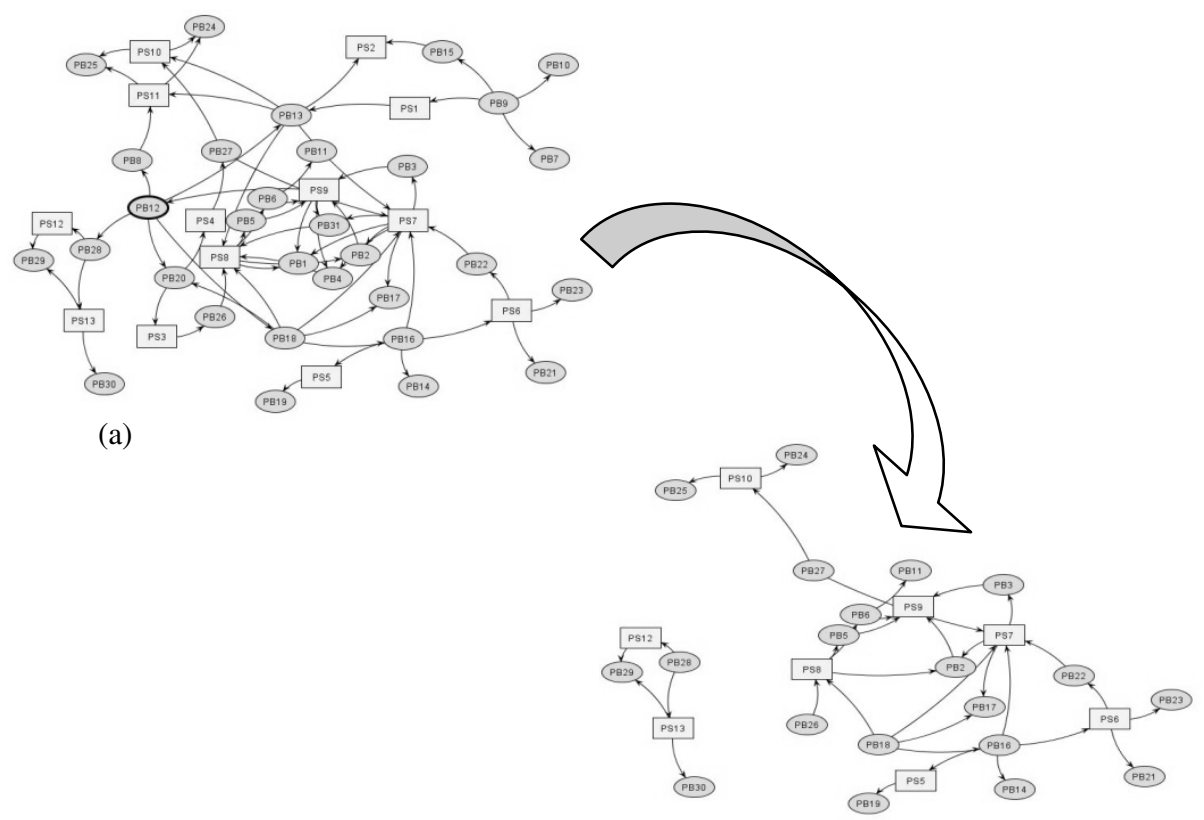

(b)

Fig. 5. Solution Concept 1.1.2.1.2 impact: from initial problem graph (a) to shrinkage after solved problems removal (b)

\section{Discussions}

After the presentation of our methodology, a question arises: To which extents a software supporting this approach can substantially find its place among a panel of already legitimated R\&D computer tools?

In the study cases we treated, the answer came from the companies' project leaders themselves. A chosen solution concept, illustrated by a scheme sometimes drawn by hand, is a good start for dimentionning, calculating and for provoking a better 
perception of the SC's technological feasibility. Therefore, calucation softwares (such as Abacus, Femlab, Comsol Multiphysics, fluent, etc...) are naturally used for further dimentionning of SCs. Traditionnal CAD systems are then naturally handeling the rest of the virtual construction of the system. Sometimes iterations are necessary with the solution concept due to calculation results. It shoud be percieved as a further clarification and fine tuning of the SC rather than of a new concept generation.

Regarding a possible integration, we don't see any evident interest in integrating the whole approach into a CAD environnement. The necessary time for conducting a study is rather long and would certainly complexify the system. As an example, the system "Product Function Optimizer" briefly introduced into CATIA V5.0 has been of an average marketing interest. Nevertheless the file resulting from our prototype of software TRIZAcquisition V3.4 are in xml format and would disserve to be placed into global PLM documentation pipeline of any project.

A complex problematic such as the one presented in this article took tree days for its problem graph constitution. Since it represents only the first part of a problem formulation stage, we faced here a difficulty related to company's practices: to allow a consequent amount of time for a task usually very brief within actual practices.

Another limit is the difficulty some managers are having to understand the role undertaken by TRIZ within results obtentions. If the developed solution concept is rather simple to understand and is a known element of any field covered by the company, they might think they could have had this idea without TRIZ. Then, the time and R\&D effort spent is even more complicated to justify. Such situations are due to lack of understanding that TRIZ is a convergent process. Unlike brainstormingoriented approach, our problematic has thouroughly evolve (its re-formulation and understanding) to avoid costly trial-and-errors. But to be able to understand this, there is a necessity to spend at least two or three days for TRIZ comprehension, its basic concepts and operating mode. This required time is rarely made available by deciders for TRIZ understanding; as a result its added value is much less percieved.

\section{Conclusions}

Is what we presented in this article something that can be called Computer Aided Innovation? The answer will certainly be more precise when a precise definition of CAI will be adopted by our community. Until now and from our understanding, CAI covers the whole innovation pipeline and can be associated to any computer tool contributing to its formalization. This undoubtedly leads CAI community to cover a so wide variety of research fields that almost any industrial aimed computer tool can fit into. On the other hand, if we reduce CAI to new tools covering inventive problems and tools assisting idea generation for inventing, it is reducing the field to a very limited amount of contributions, even too much to constitute a community. As a result we still have fuzzy boundaries to define the scope of our contributions. Nevertheless, proposing software evolutions or new softwares aiming at better serving innovation pipeline is a necessity and will anyhow be self regulated by engineers if they obviously find a relevant assistance in their proposed functionnalities regarding their needs [9]. This evolution is corelated to the evolution of the nature of the tasks they are responsible of. Throughout these evolutions, we can observe a dramatic growth of knowledges 
associated to a project, more precisely a necessity to cover a wider distance between different domains' knowledges within projects. In our article, we started with the postulate that such evolutions are undoubtedly provoking the necessity to enhance the robustness of problem understanding and formulation phases. But our thinking modes need also to change as we are ever more enclined, in complex and innovatively oriented projects to use, for solving, elements of knowledge that are unknown to us.

\section{References}

1. Miller, W., Morris, L.: Generation R\&D Managing Knowledge, Technology, and Innovation. John Wiley \& Sons, Chichester (1999)

2. Womack, J., Jones, D.T., Roos, D.: The Machine that Changed the World: Based on the Massachusetts Institute of Technology \$5 million 5-year study on the future of the automobile. Rawson, New York (1990)

3. Altshuller, G.S.: To Find an Idea: Introduction into the Theory of Inventive Problem Solving, Nauka, Novosibirsk (1986) (in Russian)

4. Cavallucci, D., Leon, N.: Towards "inventiveness-oriented" CAI tools. In: WCC 2004 (IFIP 18th World Computer Congress), pp. 441-452. Kluwer Academic Publishers, Toulouse (2004)

5. Cavallucci, D., Rousselot, F., Zanni, C.: On contradiction clouds. In: 8th ETRIA's International TRIZ Future Conference (TFC 2008), Twente, The Netherland, November 5-7 (2008)

6. Cavallucci, D., Khomenko, N.: From TRIZ to OTSM-TRIZ: Addressing complexity challenges in inventive design. International Journal of Product Development (IJPD) 4(1-2), 4-21 (2007)

7. Cavallucci, D., Rousselot, F.: Evolution Hypothesis as a means for linking system parameters and laws of engineering system evolution. In: 7th ETRIA's International TRIZ Future Conference (TFC 2007), Franckfurth, Germany, November 6-8 (2007)

8. Miles, J.C., Moore, C.J., Hooper, J.N.: A structured multi expert knowledge elicitation methodology for the development of practical knowledge based systems. In: IEE Colloquium on Knowledge Engineering, May 15, pp. 6/1-6/3 (1990)

9. Rudman, C., Engelbeck, G.: Lessons in choosing methods for designing complex graphical user interfaces. In: Rudisill, M., Lewis, C., Polson, P.B., Mc Kay, T.D. (eds.) Human Computer Interface Design: Success Stories, Emerging Methods, Real-World Context, pp. 198-228. Morgan Kaufmann, San Francisco (1996) 